\title{
IF-CONSUMO: SISTEMA DIDÁTICO PARA ANÁLISE DE CONSUMO DE ENERGIA ELÉTRICA E AUMENTO DA EFICIÊNCIA ENERGÉTICA EM INSTALAÇÕES.
}

DOI: 10.37702/2175-957X.COBENGE.2021.3739

André Roger Rodrigues - andre.roger@ifmg.edu.br

IFMG

Rua São Luiz Gonzaga, s/no , s/n

35577-010 - Formiga - MG

Paloma Maira de Oliveira - paloma.oliveira@ifmg.edu.br

IFMG

Rua Padre Alberico s/n

35577-020 - Formiga - MG

Carlos Renato Borges dos Santos - santoscrb@gmail.com IFTM

Rua Lourdes Rodrigues 157

35574-640 - Formiga - MG

Ana Flávia Peixoto de Camargos - anaflavia@ifmg.edu.br IFMG

Rua Padre Alberico 440

35577-020 - Formiga - MG

Jullia Gonçalves Lima - jullialg98@gmail.com

IFMG

rua padre alberico 440

35577-010 - Formiga - MG

Leandro Pinheiro - leandropinheiro98@gmail.com

IFMG

rua padre alberico 440

35577-010 - Formiga - MG

Resumo: Os sistemas elétricos de potência possuem regulamentação e 
normalização apropriada para sua operação de forma eficiente e confiável, tanto nos setores de geração, transmissão e distribuição de energia elétrica. Entretanto, no nível do consumidor final observamos a ausência de ações efetivas para o uso consciente e racional da energia elétrica. Este artigo apresenta uma solução que consiste na inserção de sistemas especializados na formação de técnicos e engenheiros que atuarão como agentes para disseminar o uso de tais ferramentas para população em geral, promovendo a eficiência energética nas instalações elétricas. O sistema IF-CONSUMO possui uma interface web de fácil utilização, acessivel por qualquer usuário com dispositivo conectado à internet, e que fornece orientações técnicas de fácil entendimento e implementação para que o consumidor obtenha a redução de gastos com energia elétrica. Para tanto o sistema calcula o consumo mensal e informa ao usuário modos mais eficientes de utilização dos diversos equipamentos elétricos do consumidor por meio de gráficos e de um ranking dos eletrodomésticos por consumo energético. Também é fornecida uma estimativa do valor em reais do consumo de energia baseada na maneira de utilização dos aparelhos pelo usuário. Os resultados de um estudo de caso tratando de uma residência real demonstram a eficácia do sistema quanto a estimativa do consumo de energia e do valor da conta de energia elétrica. A adoção de novos hábitos baseados nas recomendações do sistema resulta na redução efetiva de gastos com a conta de energia elétrica promovendo um uso otimizado das redes elétricas.

Palavras-chave: Consumo de energia elétrica. Eficiência energética. Soluções em software. Ensino com sistemas inteligentes. 


\section{(C) COBENGE \\ 28 a 30 de SETEMBRO \\ IF-CONSUMO: SISTEMA DIDÁTICO PARA ANÁLISE DE CONSUMO DE ENERGIA ELÉTRICA E AUMENTO DA EFICIÊNCIA ENERGÉTICA EM INSTALAÇÕES.}

\section{INTRODUÇÃO}

A necessidade de economia de energia elétrica é o motivo principal para o estabelecimento de ações socioeconômicas e políticas que almejam a construção de uma consciência ambiental para o uso racional dos recursos energéticos primários pela população. Tal fato levou a grandes esforços da comunidade científica para a pesquisa e o desenvolvimento de sistemas e ferramentas auxiliares que proporcionem o uso mais eficiente da energia elétrica. Entretanto é no nível do consumidor final em baixa tensão, que representa $26 \%$ de todo o consumo de energia elétrica brasileiro (EPE, 2020), onde podemos notar a ausência de ações mais efetivas para o uso consciente e eficiente da energia elétrica. Segundo o atlas de eficiência energética (EPE, 2020), uma parcela de $16,2 \%$ de toda energia produzida no país é perdida. Tal fato evidencia a carência de sistemas e ferramentas acessíveis a população em geral, e que seja obrigatoriamente de fácil utilização, voltados a redução de gastos do consumidor com a conta de energia elétrica e, adicionalmente, contribua para uma utilização da energia elétrica de maneira mais eficiente (DARBY, 2008). O entendimento é que a redução das perdas de energia elétrica observadas no nível do consumidor de baixa tensão é dependente da popularização do uso de ferramentas especialistas que forneça orientações práticas e que sejam facilmente implementadas pelo consumidor em geral. A disseminação de uso de tais sistemas se consolidará a partir do momento em que os futuros profissionais do setor elétrico, técnicos e engenheiros, incorporem estas soluções em seus projetos e, principalmente, apresentem tais recursos à cadeia de clientes e empresa ao longo da prestação de seus serviços, atingindo assim o consumidor final. Diante do exposto este trabalho apresenta uma solução inteligente em software, que permite realizar a estimativa do consumo de energia de uma residência e fornece recomendações práticas e informações ao usuário destinadas a adoção de novos hábitos acerca da utilização mais eficiente e correta de sua instalação elétrica e dos equipamentos eletroeletrônicos nela utilizados.

Especificamente, o sistema desenvolvido consiste na coleta de dados acerca da rotina de uso dos aparelhos elétricos e eletrônicos do usuário, a partir dos quais é realizada uma análise do perfil do consumidor. Os dados de entrada são processados e informações técnicas e recomendações práticas são fornecidas para o apoio quanto a tomada de decisão pelo usuário em tornar-se um consumidor de energia elétrica mais eficiente e ambientalmente consciente.

\section{REFERENCIAL TEÓRICO}

Uma das formas de reduzir as perdas é através de programas de conscientização dos consumidores. O consumo consciente está associado à utilização racional de energia elétrica, onde através de pequenas mudanças de hábitos praticadas pelo indivíduo em sua residência, evita-se o desperdício de eletricidade, poupando recursos nos mais diversos processos de produção de energia (FOURRIER, 2010). A capacidade de monitorar gastos e identificar os pontos mais críticos é um dos encargos para um desenvolvimento sustentável, caracterizando uma ferramenta estratégica para diminuição das contas de 
energia elétrica. ALAHMAD et al. (2012) afirma que, o acesso as informações coletadas da rede elétrica auxiliam com uma redução do consumo energético, pelo próprio consumidor, entre $10 \%$ e $20 \%$. Esta é característica fundamental das redes elétricas inteligentes, mas que atualmente não está disponível ao consumidor final que está conectado ao sistema elétrico por meio de uma topologia clássica da rede de distribuição secundária. Neste sentido atualmente são identificados alguns trabalhos desenvolvidos com base na utilização de hardware, tais como, Arduino e/ou Raspberry (KHEDKAR, 2016), que é por vezes eficaz proporcionando uma boa precisão na coleta dos dados. Contudo, esta é uma solução ainda incipiente para os consumidores em baixa tensão no Brasil. Alguns simuladores de consumo foram desenvolvidos por concessionárias de energia, tais como a COPEL, a LIGHT, a ENEL. Em geral, estas aplicações são tecnologicamente limitadas quanto comparadas ao sistema do IF-CONSUMO uma vez que são aplicações web. Em nenhum destes simuladores online há algoritmos especializados para orientação do usuário quanto a potência elétrica correta de seus equipamentos. Também não foram identificados simuladores que tenham as funcionalidades tais como a geração do relatório técnico com ranking de equipamentos e elaboração de gráficos que identificam claramente quais os equipamentos estão elevando o consumo de energia elétrica. A COPEL (Companhia Paranaense de Energia) desenvolveu um simulador de consumo residencial, comercial e rural. O usuário informa os aparelhos de sua residência e a frequência de uso dos mesmos. O simulador retorna o consumo de energia em kWh e uma estimativa do valor da conta de energia. Nenhuma recomendação para economia de energia é fornecida. Obviamente não é possível realizar nenhum cadastro neste simulador. O simulador de consumo da Light, possui uma separação de equipamentos por categorias embora seja um sistema muito simples onde o usuário seleciona os aparelhos presentes em sua residência e o sistema fornece uma potência estimada. O resultado é fornecido em forma de tabela, de maneira elementar sem qualquer recomendação para reduzir o consumo de energia. O simulador da Enel é um dos simuladores mais completos e eficientes dentre aqueles identificados durante a revisão bibliográfica. Este possui um sistema de fácil utilização. O usuário informa os equipamentos presentes em sua residência e o sistema fornece uma potência estimada. Após a análise é gerado um relatório com gráficos e recomendações de mudanças de hábitos, entretanto as recomendações oferecidas não são práticas, uma vez que, é sugerido ao usuário a troca do equipamento, representando um alto custo para o pequeno consumidor adotar as soluções sugeridas. Não há possibilidade de realização de cadastros de usuários, equipamentos, fabricantes, unidades consumidoras ou cômodos.

\section{METODOLOGIA}

O desenvolvimento do sistema exigiu a integração de áreas de conhecimento relacionadas tanto a Engenharia Elétrica quanto a Ciência da Computação devido ao caráter multidisciplinar inerente ao sistema. A metodologia empregada para desenvolvimento do sistema proposto foi definida por etapas que são descritas a seguir.

\subsection{Levantamento de dados e especificações dos aparelhos eletroeletrônicos}

Inicialmente foi realizado um levantamento acerca dos equipamentos elétricos comumente utilizados por um consumidor residencial típico, classificando-os em categorias específicas de equipamentos eletroeletrônicos que possuem características elétricas semelhantes, tais como aquecimento, máquinas motrizes, iluminação, etc. Assim sendo o sistema IF-CONSUMO já possui em sua base de dados um conjunto predeterminado de 
aparelhos eletroeletrônicos que estão comumente presentes nas instalações elétricas de consumidores residenciais. Nesta etapa foram catalogadas as especificações elétricas necessárias para cálculo da energia elétrica consumida pelos aparelhos eletroeletrônicos, tais como potência elétrica, tensão de alimentação, além das dimensões, do modelo do equipamento e demais informações técnicas fornecidas pelos fabricantes. A pesquisa bibliográfica realizada nesta etapa evidenciou que as potências elétricas dos aparelhos variam muito entre diferentes fabricantes. Como solução IF-CONSUMO adotou os valores de potências elétricas comerciais de aparelhos informadas nos relatórios do Programa de Análise de Produtos (INMETRO, 2020). Com a incorporação destas informações técnicas dos relatórios do INMETRO no banco de dados, o IF-CONSUMO se consolida como uma ferramenta que contribui para o fortalecimento e disseminação do Programa Brasileiro de Etiquetagem (PBE) que fomenta a eficiência energética dos eletrodomésticos, ao permitir ao usuário comprovar a economia de gastos com energia elétrica ao optar por um aparelho mais eficiente na hora da compra ou troca de aparelhos antigos, com base nos resultados de processamento do sistema. Também foram catalogados dados de aparelhos obtidos em documentos técnicos do programa Energy Star (ENERGYSTAR, 2020) que consiste em uma certificação de eficiência energética adotada por diversas nações e por fabricantes nacionais e internacionais. Por último foi realizada também a catalogação de outros aparelhos eletroeletrônicos de diferentes fabricantes ou capacidades que eventualmente não constavam nestes relatórios citados, mas que são comercializados em nosso país. A Figura 1 apresenta uma interface gráfica exemplificando alguns dos aparelhos pré-cadastrados. Para seleção/edição de um aparelho há diversos filtros tais como Aparelho, Fabricante, Potência, Modelo, Categoria, por meio de uma lista suspensa.

Após realizar o login no sistema IF-CONSUMO o usuário pode acessar o menu mostrado a esquerda na Figura 1. Ao clicar na opção Cadastros Diversos são visualizados os aparelhos previamente cadastrados. Novos cadastros e edições podem ser realizados pelo usuário nas opções Categorias, Cômodos, Marcas, Usuários ou Residências do menu.

Figura 1 - Interface com exemplos de aparelhos eletroeletrônicos pré-cadastrados.

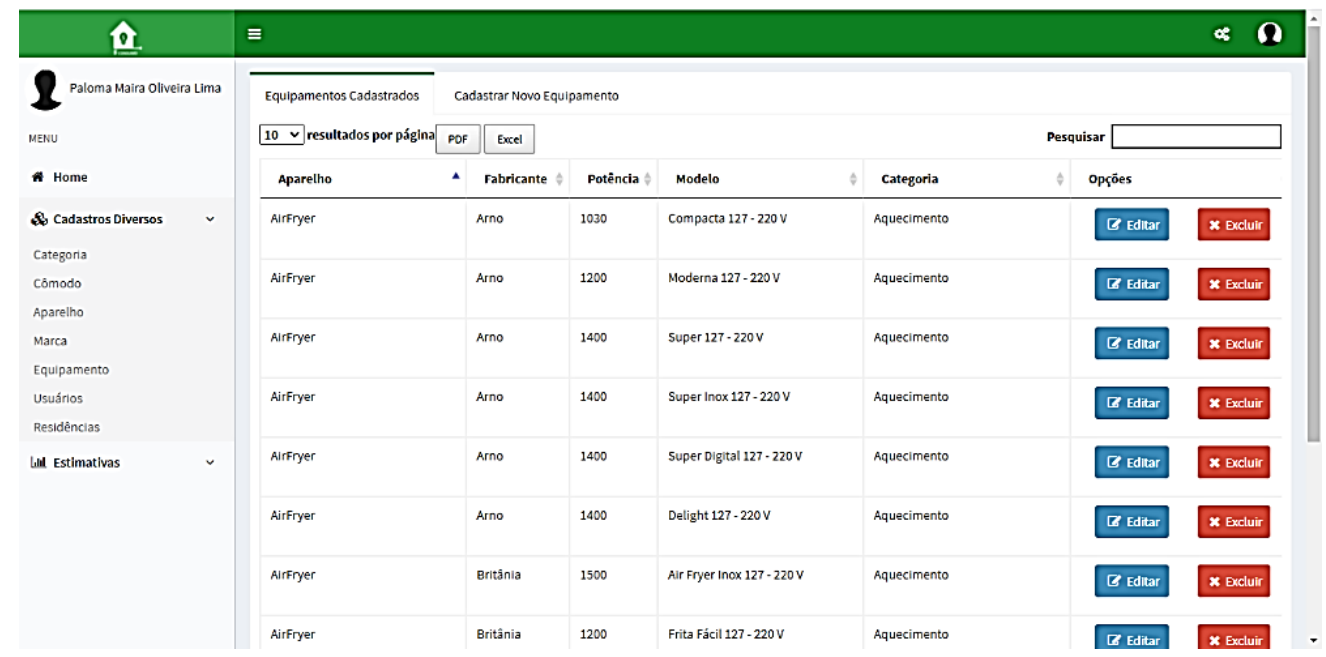

Fonte: Elaborada pelos autores

A Figura 2 exibe a interface para cadastrar novos equipamentos.

Para desenvolvimento do sistema IF-CONSUMO foi necessário elaborar procedimentos específicos para determinação da potência elétrica de aparelhos tais como televisores, condicionadores de ar e iluminação. Tais procedimentos foram determinados 
também com objetivo de tornar o IF-CONSUMO em um sistema com interface amigável e passível de ser utilizado por qualquer tipo de usuário, seja um profissional da área de elétrica ou um consumidor comum que não tem nenhum conhecimento específico. Esta observação acabou por determinar a criação de dois tipos de usuários para o sistema do IF-CONSUMO, podendo se um usuário básico ou especialista.

Figura 2 - Interface para cadastrar novos aparelhos eletroeletrônicos.

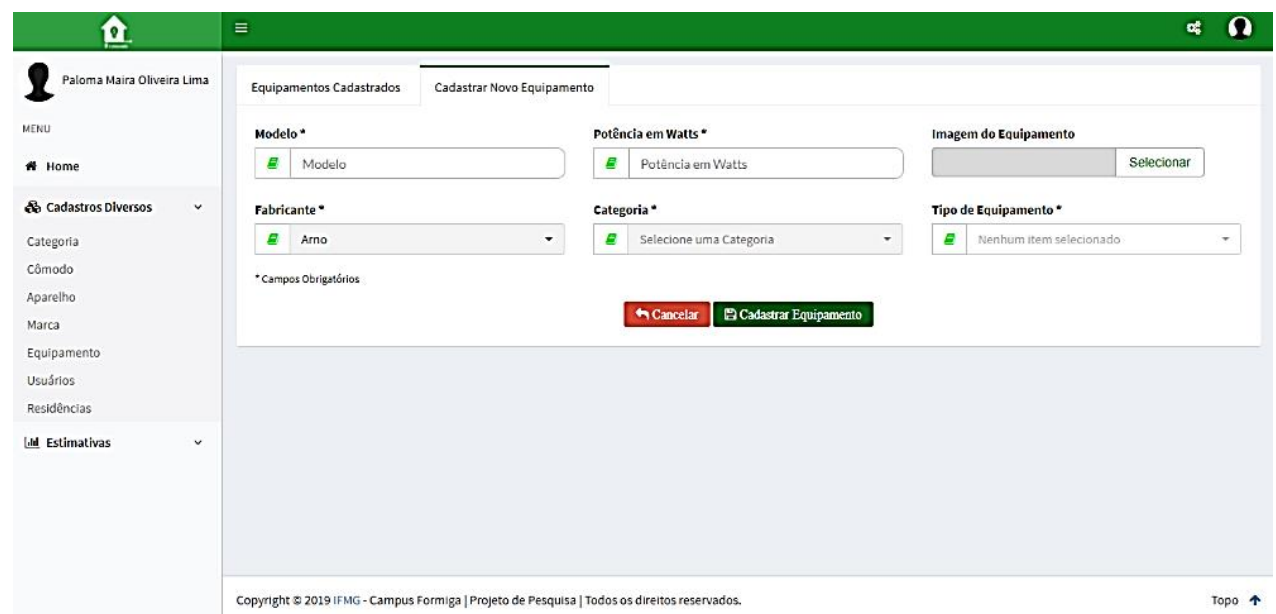

Fonte: Elaborada pelos autores

A Figura 3 apresenta os usuários cadastrados com atribuição do perfil de usuário básico ou especialista. O usuário básico é aquele que desconhece as potências elétricas e demais especificações de seus aparelhos e não têm condições de informar corretamente os dados de entrada no sistema. Já o usuário especialista tem conhecimentos na área de elétrica e é capaz de determinar com precisão todas as especificações elétricas dos aparelhos seja por inspeção visual do aparelho, por consulta aos manuais de fabricantes e/ou sites, publicações e catálogos especializados. O especialista está apto a cadastrar novos equipamentos e conhece a potência e as características de seus aparelhos. Esta interface apresenta também a opção Cadastrar Novo Usuário.

Figura 3 - Interface para cadastrar novos usuários

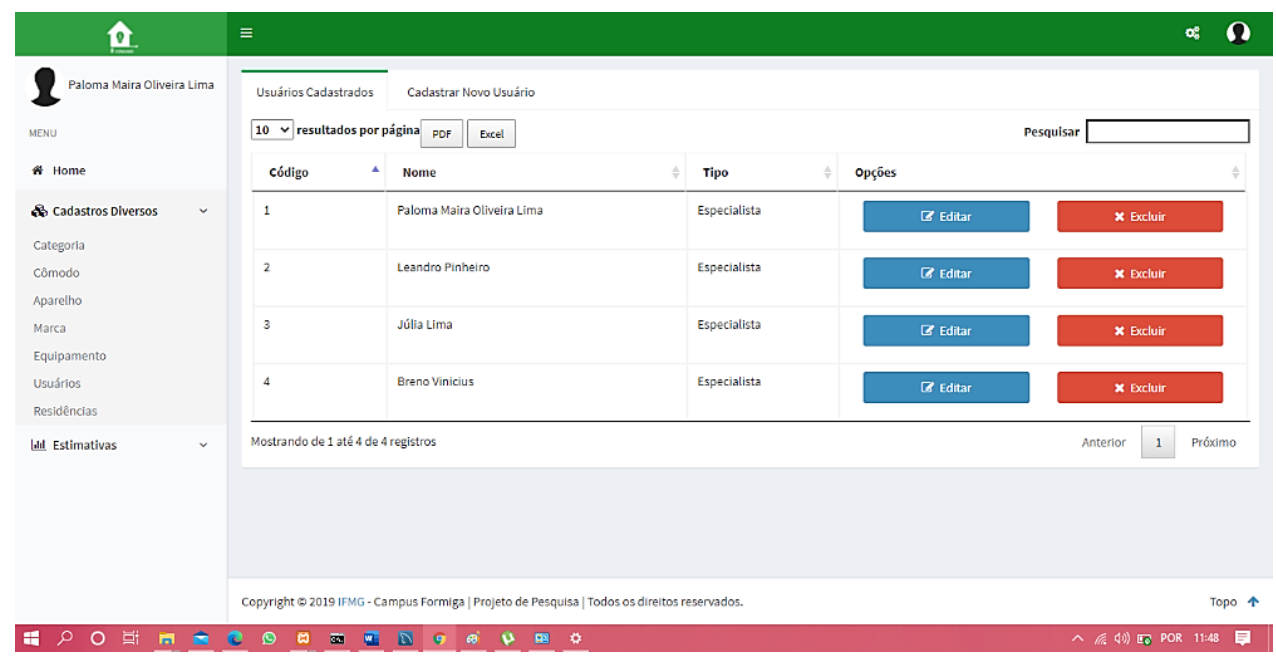

Fonte: Elaborada pelos autores 
Tanto o usuário básico quanto o usuário especialista, podem clicar em uma lista suspensa e informar corretamente a potência elétrica do seu televisor, caso a conheçam. Entretanto, para o usuário básico, que eventualmente não conhece ou não tem como determinar a potência elétrica do seu aparelho por falta de conhecimentos específicos em elétrica, o sistema IF-CONSUMO já fornece um recurso adicional para determinar a potência elétrica do aparelho televisor de maneira bem simples, bastando que o usuário informe a medida da diagonal da tela do aparelho televisor e se o televisor é do tipo tela plana ou tubo.

\subsection{Implementação do banco de dados do IF-CONSUMO}

A etapa seguinte foi dedicada a criação de um banco de dados incluindo os valores de potência elétrica de cada um dos equipamentos elétricos elencados na primeira etapa. Esta ação foi necessária para realização de cálculos para estimativa da energia elétrica consumida por cada equipamento considerado, expressa em kWh. O banco de dados contém todas estas especificações elétricas necessárias agrupadas por fabricante, por modelo ou até dimensões e capacidade para cada um dos aparelhos eletroeletrônicos précadastrados. Já para a persistência dos dados foi utilizado o banco de dados MYSQL que é um sistema gerenciador de banco de dados relacional que possui código aberto (MILANI, 2007).

A criação deste banco de dados permitiu a implementação de outra funcionalidade específica do sistema IF-CONSUMO não encontrada em sistemas similares. Para a definição da potência elétrica de cada aparelho, o usuário pode clicar em uma lista suspensa onde é possível selecionar valores específicos de potência elétrica deste tipo de aparelho classificados por modelo ou por fabricante. O usuário também pode escolher um valor médio entre os valores de potência elétrica de diferentes fabricantes. Este recurso é particularmente importante para os casos nos quais não usuário não tem certeza da potência de seus aparelhos. Este valor médio baseado nos valores de potência de diferentes fabricantes foi chamado de "Smart Media" e está baseado nos valores de potências elétricas extraídos dos relatórios do INMETRO e catalogados no banco de dados do IF-CONSUMO.

Figura 4 - Interface para entrada de dados de aparelhos televisores

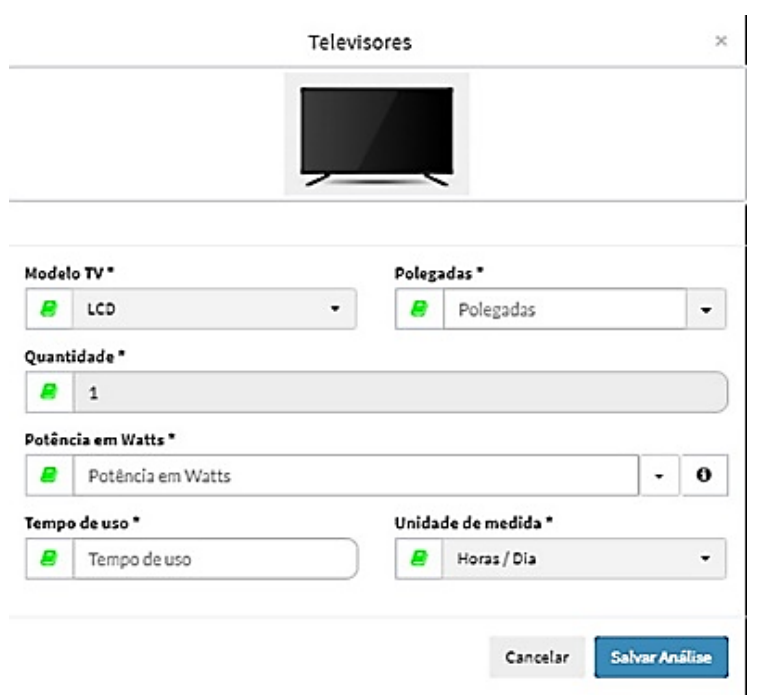

Fonte: Elaborada pelos autores 


\subsection{Registro e exibição de recomendações práticas que promovam a} redução dos gastos com energia elétrica

$\mathrm{Na}$ terceira etapa foram analisadas e documentadas as principais características técnicas dos equipamentos pré-cadastrados. Foram também registrados os maus hábitos de uso dos eletrodomésticos para diferentes categorias que determinam o aumento do valor gasto com a conta de energia elétrica. A definição dos maus hábitos de utilização dos equipamentos adotou critérios específicos para diferentes categorias de aparelhos, tais como o horário de uso, a frequência de uso, o tipo do equipamento e sua utilização observando-se a rotina diária dos consumidores, aparelhos de aquecimento, de climatização de ambientes, aparelhos com motores e iluminação.

\subsection{Análise e Modelagem do sistema IF-CONSUMO}

A quarta etapa destinou-se a modelagem da interface WEB incorporando as melhores características de sistemas similares já existentes e que foram identificados na revisão bibliográfica, aprimorando-as. Para a modelagem foram utilizados fluxogramas e modelos mentais tal como mostrado na Figura 4, com o intuito de proporcionar melhor visualização e compreensão acerca da forma como os diferentes objetos se comunicam. Foi necessário desenvolver tais modelos para aparelhos televisores, condicionadores de ar e iluminação. Os fluxogramas e modelos mentais também descrevem as principais funcionalidades do sistema e a interação dos mesmos. Nesta etapa também foram realizados estudos e análises sobre a melhor maneira de como o sistema IF-CONSUMO deveria exibir as recomendações práticas ao usuário, para cada tipo de equipamento, com intuito de reduzir os gastos com energia elétrica. Também foi desenvolvida a interface gráfica (camada de visão) além do back-end (camada controle e modelo). Para a definição da interface gráfica do sistema (front-end) foram utilizadas as seguintes tecnologias:

i. HTML que é uma linguagem de marcação bastante utilizada na construção de páginas na web (DUCKETT, 2015).

ii. CSS: trata-se de uma linguagem de folhas de estilo comumente usada para definir a apresentação de documentos escritos em uma linguagem de marcação, normalmente HTML [DUCKETT, 2015].

iii. JavaScript: é uma linguagem interpretada, que inicialmente foi projetada como uma parte de navegadores web, pois assim os scripts podem ser executados pelo cliente sem que seja preciso passar pelo servidor [FLANAGAN, 2004].

iv. Bootstrap: consiste de uma biblioteca de front-end com código aberto para a criação de sites e aplicações web [BHATT; SINGH, 2016].

Para a implementação do back-end foi utilizada a linguagem PHP que é uma linguagem interpretada livre que inicialmente era destinada para o desenvolvimento de aplicações que estão presentes e atuam na área do servidor, que geram conteúdo dinâmico na web (THONSON; WELLING, 2005) e o framework para PHP Laravel ao qual utiliza arquitetura MVC (modelo, visão e controle) (LARAVEL, 2017). Desenvolvimento de aplicações seguras e com boa performance de modo rápido, com código simples e limpo, caracterizam as principais características desse sistema. Por fim foi realizada a integração entre as camadas ao final desta etapa.

3.5 Geração de relatório geral de análise do consumo residencial e recurso gráficos para exibição de resultados

$\mathrm{Na}$ última etapa foram implementados os recursos de geração de um relatório geral pós processamento do sistema a fim de informar ao consumidor os gastos estimados de 
sua residência, em $\mathrm{kWh} / \mathrm{mês}$ e em reais. Estes relatórios contém uma estimativa de consumo e também os resultados da análise feita pelo sistema. O sistema exibe os resultados de análises de consumo de energia elétrica do usuário em formato gráfico e/ ou em formato pdf.

\section{RESULTADOS}

Nesta seção são apresentados os resultados de um estudo de caso realizado com a finalidade de validação dos procedimentos de cálculo do sistema IF-CONSUMO. Adicionalmente são também apresentadas as suas principais funcionalidades do sistema aplicadas tanto a estimativa de consumo de energia elétrica quanto a economia de gastos com energia elétrica por adoção das recomendações práticas em favor do aumento da eficiência energética da instalação. O estudo considera uma unidade consumidora residencial, habitada por 2 pessoas. O levantamento de dados e frequência de uso dos eletrodomésticos estão resumidos no Quadro 1.

Os dados de potência elétrica e frequência de uso dos eletrodomésticos foram inseridos no sistema IF-CONSUMO obtendo-se como resultado um consumo de energia elétrica total de $259,27 \mathrm{kWh} / \mathrm{mês}$. A Figura 5 apresenta alguns recursos gráficos para exibição dos resultados de consumo detalhados para cada cômodo tanto para aparelhos quanto por iluminação. O IF-CONSUMO também exibe um gráfico em formato de pizza que evidencia o consumo por aparelhos e por categorias de aparelhos.

Quadro 1 - Dados para estimativa de consumo de energia elétrica de uma residência.

\begin{tabular}{|c|c|c|c|c|c|c|}
\hline \multicolumn{7}{|c|}{ Levantamento de dados para estimativa do consumo de uma residência } \\
\hline Cômodos & Aparelhos & Potência (watts) & Tempo (horas) & Tempo (min) & Dias & Consumo \\
\hline Cozinha & Geladeira & 135 & 16 & 0 & 30 & 64,80 \\
\hline & Liquidificador & 748 & 0 & 5 & 12 & 0,75 \\
\hline & Micro-ondas & 1140 & 0 & 10 & 15 & 2,85 \\
\hline & Air Fryer & 1500 & 0 & 30 & 8 & 6,0 \\
\hline & Sanduicheira & 700 & 0 & 5 & 12 & 0,70 \\
\hline & Iluminação & 23 & 4 & 0 & 30 & 2,76 \\
\hline Sala & Roteador & 15 & 24 & 0 & 30 & 10,8 \\
\hline & Recepto & 150 & 4 & 0 & 30 & 18,0 \\
\hline & Televisão & 230 & 4 & 0 & 30 & 27,6 \\
\hline & Iluminação & 49 & 2 & 0 & 30 & 2,94 \\
\hline Banheiro & Chuveiro & 5500 & 0 & 15 & 8 & 41,25 \\
\hline & Secador & 1900 & 0 & 15 & 8 & 3,80 \\
\hline & Iluminação & 23 & 2 & 0 & 30 & 1,38 \\
\hline Banheiro & Chuveiro & 5500 & 0 & 15 & 30 & 41,25 \\
\hline & Iluminação & 23 & 1 & 0 & 30 & 0,69 \\
\hline Quarto 1 & Notebook & 200 & 1 & 0 & 15 & 3,00 \\
\hline & Televisão & 51,05 & 4 & 0 & 30 & 6,13 \\
\hline & Celular & 5 & 4 & 0 & 30 & 0,60 \\
\hline & Iluminação & 23 & 5 & 0 & 30 & 3,45 \\
\hline Quarto 2 & Celular & 5 & 4 & 0 & 30 & 0,60 \\
\hline & Iluminação & 23 & 5 & 0 & 30 & 3,45 \\
\hline & Televisão & 51,05 & 4 & 0 & 30 & 6,13 \\
\hline & Ventiladores & 115 & 3 & 0 & 30 & 10,35 \\
\hline & & Consumo Total em kWh/mês & & & 259,27 \\
\hline
\end{tabular}

Fonte: Elaborado pelos autores. 
Figura 5 - Alguns dos recursos gráficos para exibição de resultados

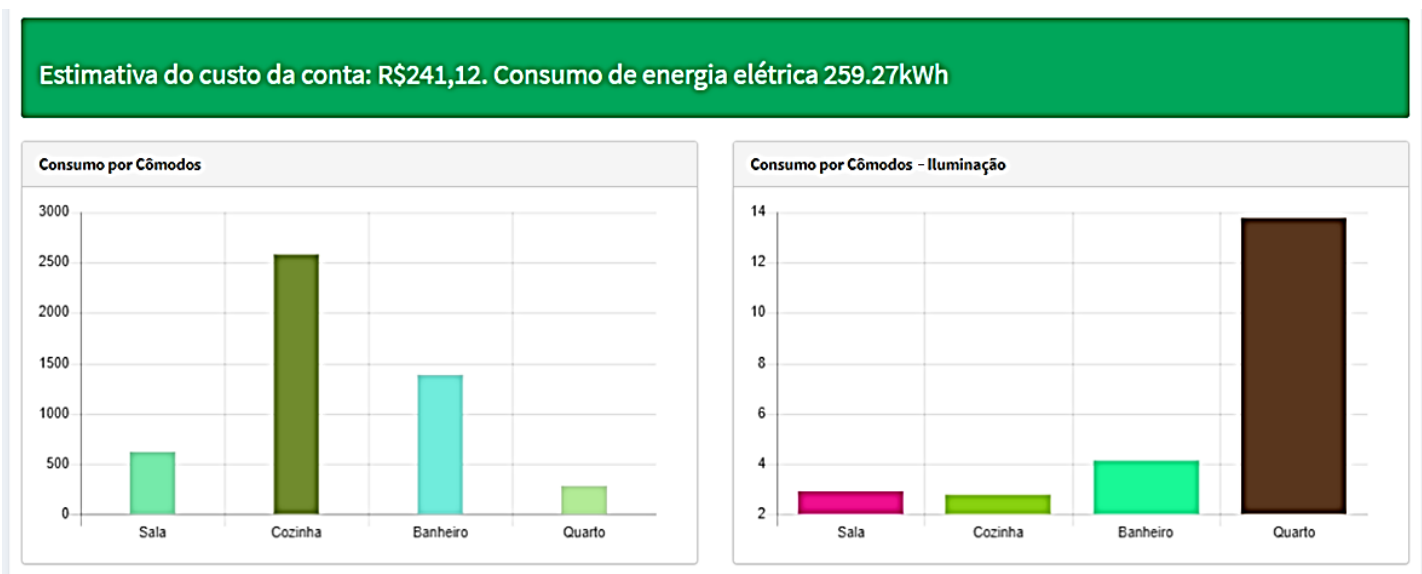

Fonte: Elaborada pelos autores

A Figura 6 apresenta a interface com dados dos aparelhos da residência lançados no sistema.

Após o processamento e cálculo da estimativa de consumo de energia o IF-CONSUMO apresenta recomendações específicas tal como a mostrada na Figura 7 para que o consumidor adote bons hábitos para redução da energia elétrica consumida.

Figura 6 - Interface para inclusão dos dados com alguns dos aparelhos do Quadro 1.

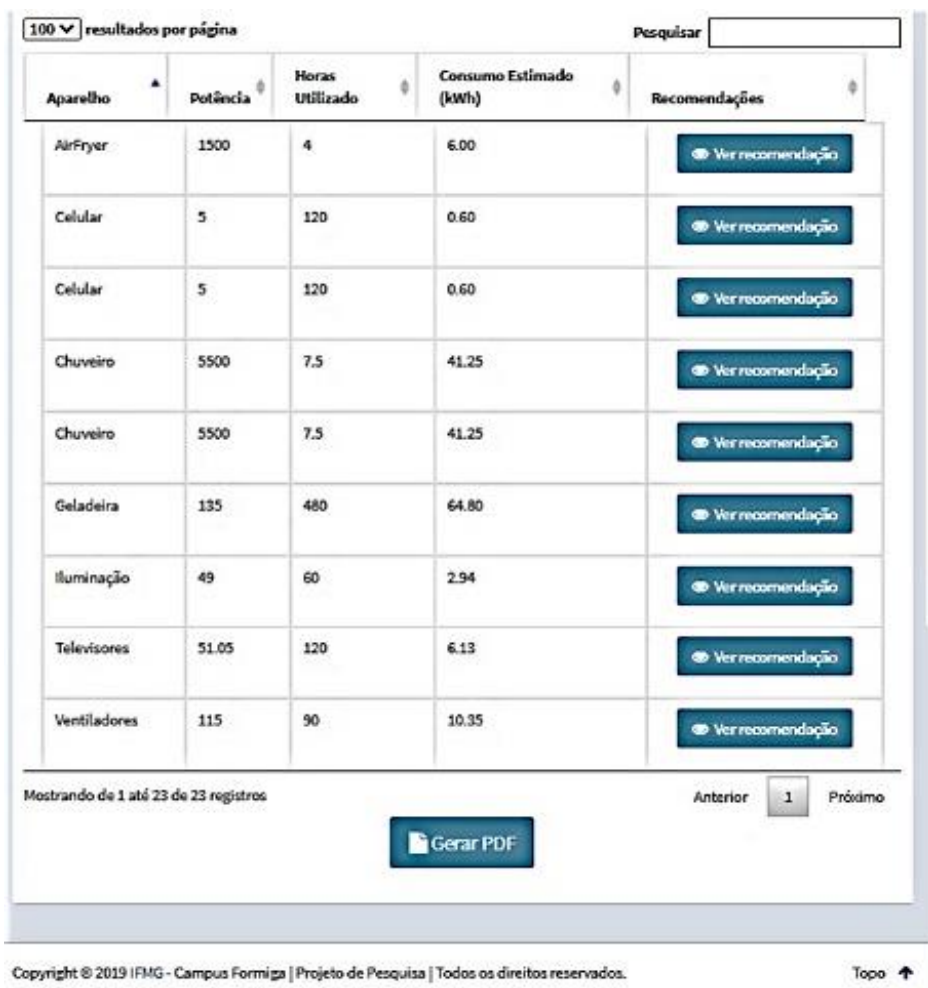

Fonte: Elaborada pelos autores

A Figura 8 apresenta o consumo de energia elétrica com valor de $253 \mathrm{kWh} / \mathrm{mês}$ em agosto de 2020 e de $22 \mathrm{kWh} / \mathrm{mês}$ em setembro de 2020. 
Figura 7 - Exemplo de recomendações práticas do

IF-CONSUMO para economia de energia elétrica.

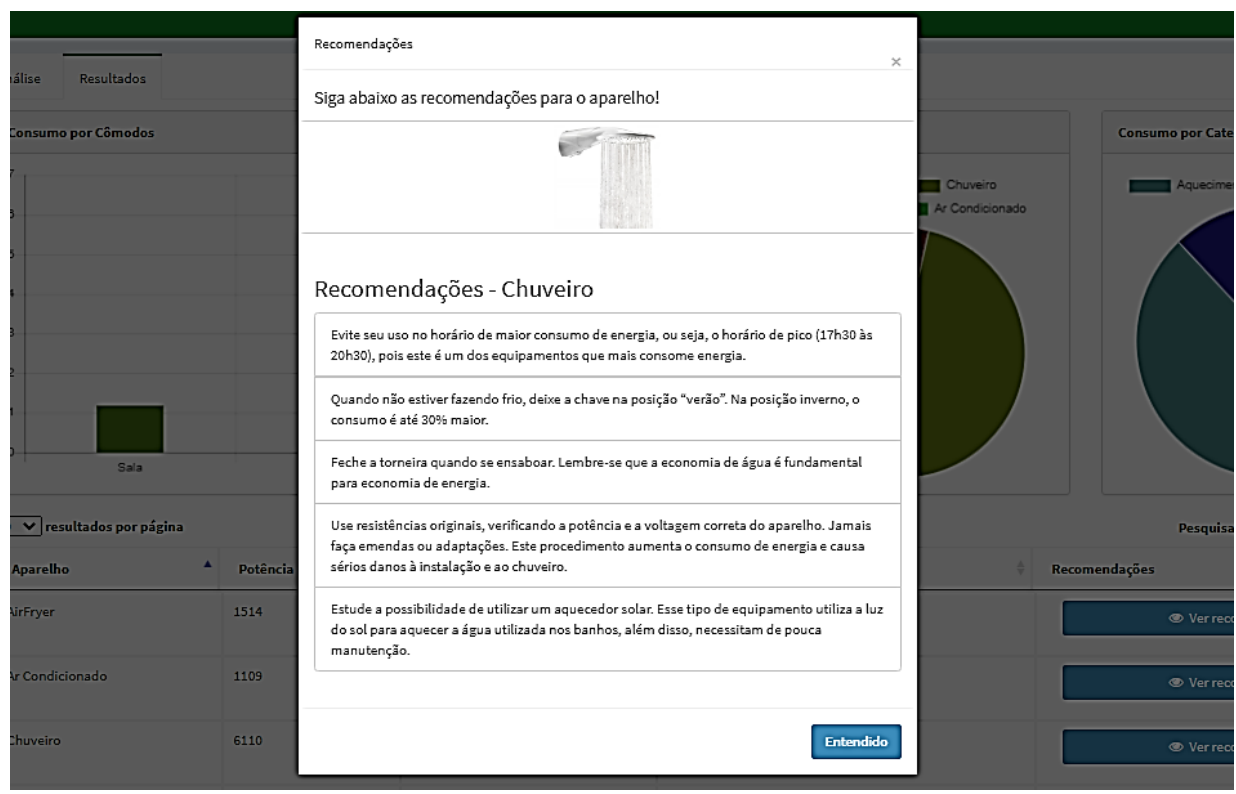

Fonte: Elaborada pelos autores

Após os moradores adotarem as recomendações sugeridas pelo IF-CONSUMO, tais como alteração da temperatura do chuveiro da posição INVERNO para a posição VERÃO, a substituição das lâmpadas incandescentes por lâmpadas LED e as recomendações práticas dos demais equipamentos, obtém-se uma redução de consumo para $206 \mathrm{kWh} / \mathrm{mês}$ em outubro.

Figura 8 - Consumo de energia elétrica registrados em 3 meses.

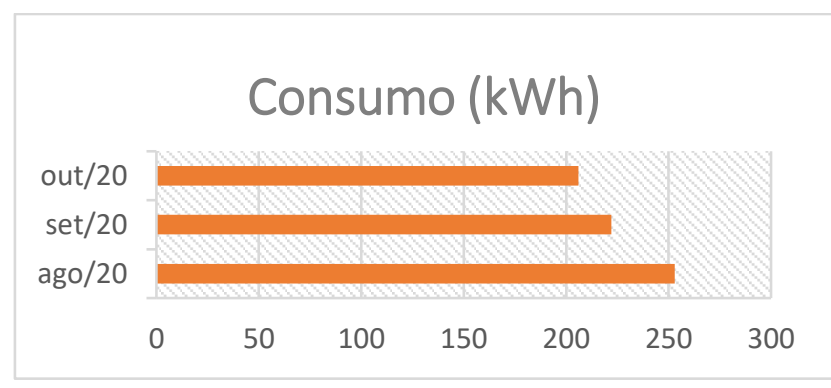

Fonte: Elaborada pelos autores

A Figura 9 apresenta as contas de energia elétrica para a unidade analisada para os meses de agosto, setembro e outubro de 2020, comprovando-se neste a redução do consumo de energia elétrica.

Figura 9 - Contas de energia elétrica do usuário.

a) Conta de Agosto de 2020.

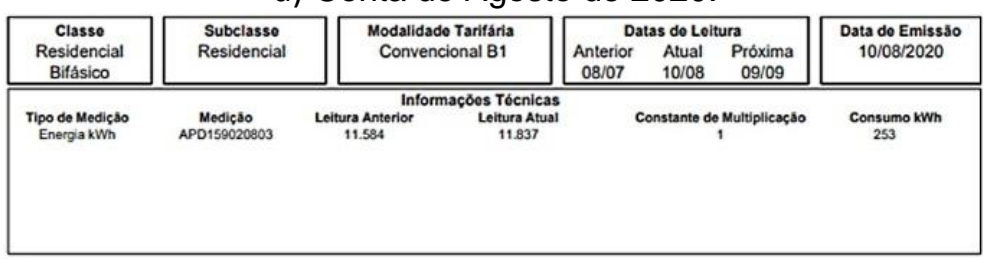


b) Conta de Setembro de 2020.

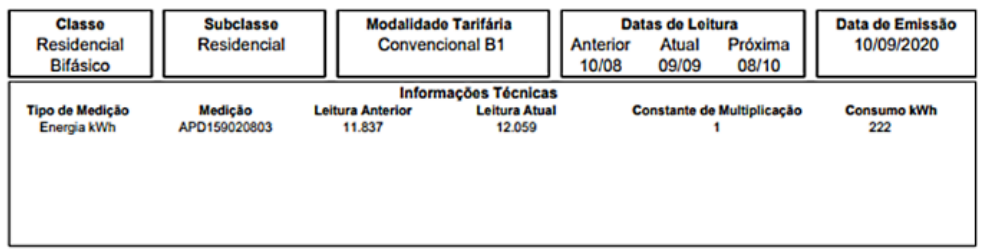

b) Conta de Outubro de 2020.

\begin{tabular}{|c|c|c|c|c|}
\hline \multicolumn{4}{|c|}{ Informaçōes Técnicas } & \multirow[b]{2}{*}{ Consumo kWh } \\
\hline Tipo de Medição & Leitura Anterior & Leitura Atual & Constanto de Mediçăo & \\
\hline Energia Elétrica & 12059 & 12265 & 1 & 206 \\
\hline \multicolumn{5}{|c|}{ VALORES FAIURADOS } \\
\hline \multicolumn{4}{|c|}{ ENCARGOS/COBRANCCAS } & 189,47 \\
\hline
\end{tabular}

Fonte: Elaborada pelos autores

Os resultados apresentados evidenciam a eficácia do simulador IFCONSUMO, uma vez que os valores de consumo obtidos pela análise estavam em conformidade com os valores extraídos das referências e também das contas de energia elétrica fornecidas pela concessionária de energia.

\section{CONSIDERAÇÕES FINAIS}

Este artigo apresentou um sistema didático com interface web, capaz de realizar a estimativa do valor do consumo de energia elétrica e calcular o valor em reais da conta de energia elétrica, a partir dos dados fornecidos pelo usuário. Adicionalmente o sistema fornece recomendações práticas voltadas para economia de energia com base na análise do consumo de energia por aparelho e por hábito de uso do consumidor.

A incorporação de recursos pedagógicos tal como o sistema especializado IF-CONSUMO nas disciplinas de cursos técnicos da área de energia elétrica e de cursos de Engenharia é de fundamental importância para disseminação destas tecnologias para o consumidor em geral com intuito de combater o desperdício e as perdas de energia elétrica nas unidades consumidoras em baixa tensão.

Os resultados comprovam que o sistema IFCONSUMO permite ao usuário identificar os equipamentos eletroeletrônicos que mais consomem energia elétrica por meio da análise dos gráficos exibidos no relatório de análise. Desta forma o sistema IFCONSUMO permite ao usuário reavaliar os seus hábitos de consumo optando por ações que efetivamente resultem na redução nos valores gastos com energia. Embora este estudo de caso envolva um consumidor residencial, esta ferramenta pode também ser utilizada por consumidores prediais, comerciais e até industriais devido a funcionalidade do IF-CONSUMO para cadastro de novos aparelhos, fornecedores e até mesmo cômodos e ambientes.

\section{Agradecimentos}

Os autores agradecem ao IFMG-Campus Formiga pelo apoio técnico e financeiro.

\section{REFERÊNCIAS}

ALAHMAD, M. A., WHEELER, P. G., SCHWER, A., EIDEN, J., BRUMBAUGH, A. A comparative study of three feedback devices for residential real-time energy 
monitoring. IEEE Transactions on Industrial Electronics, v.59, n.4, 2012, p.2002-2013. https://doi.org/10.1109/TIE.2011.2165456

BHATT, M.; SINGH, H. Learning Web Development with React and Bootstrap. [S.I.]:

Packet Publishing, 2016.

DUCKETT, J. HTML e CSS Projete e Construa Websites. [S.I.]: Alta Books, 2015.

Empresa de pesquisa energética (EPE). BRASIL. Atlas da Eficiência Energética

Brasileira. Disponível em: http://www.epe.gov.br/pt/publicacoes-dados-

abertos/publicacoes/atlas-da-eficiencia-energetica-brasil-2019. Acesso: 28 de janeiro de 2020.

Empresa de Pesquisa Energética (EPE). BRASIL. Balanço Energético Nacional 2020: Ano base 2019 / Empresa de Pesquisa Energética. - Rio de Janeiro: EPE, 2020.

EnergyStar. ENERGY STAR | The simple choice for energy efficiency. Disponível em: https://www.energystar.gov/about. Acesso em: 19 de maio de 2021.

FLANAGAN, D. JavaScript: o guia definitivo. Porto Alegre: Bookman, 2004.

FOURRIER, Anna Carolina Pires. Energia Elétrica do Setor Residencial à Luz do Consumo Consciente: Resultados do Estudo de Caso no Município de Santo André (SP). V Encontro Nacional da Anppas, 2010.

INMETRO - Instituto Nacional de Metrologia, Qualidade e Tecnologia. BRASIL. Ministério da Economia. Disponível em https://dados.gov.br/dataset/programa-de-analise-de-

produtos-pap. Acesso em 19 de maio de 2021.

KHEDKAR, S.; MALWATKAR, G. M. Using raspberry Pi and GSM survey on home automation. In: INTERNATIONAL CONFERENCE ON ELECTRICAL, ELECTRONICS, AND OPTIMIZATION TECHNIQUES (ICEEOT). Proceedings. pp 758-761, 2016. https://doi.org/10.1109/ICEEOT.2016.7754787

LARAVEL. Laravel Documentation. Disponível em: <https://laravel.com>. Acesso em: 12 de maio de 2020.

MILANI, A. MySQL-guia do programador. [S.I.]: Novatec Editora, 2007.

DARBY, S. Energy feedback in buildings: improving the infrastructure for demand reduction. Building Research \& Information, v.36, n.5, p.499-508, 2008. Disponível em: https://doi.org/10.1080/09613210802028428

THONSON, L.; WELLING, L. Php e mysql: desenvolvimento web. Rio de Janeiro: Campus, 2005.

\section{IF-CONSUMO: EDUCATIONAL SYSTEM APPLIED TO ENERGY CONSUMPTION ANALYSYS AND ENERGY EFFICIENT IMPROVEMENTS IN ELECTRICAL INSTALLATIONS}


Abstract: According to the electrical regulation and standards, the electric power systems must be operated in an efficient e reliable way, both in generation, transmission and distribution levels. Although, there are not effective actions to provide an optimized use of electrical energy at the low voltage costumers. This article presents a solution that consists of the insertion of smart systems based learning at technicians and engineers courses once these professionals will act as agents to disseminate the use of such tools among the general population, promoting energy efficiency in electrical installations. The IFCONSUMO system has an easy-to-use web interface, available by means of an internetconnected device. The application provides technical guidelines that can be easily understood and implemented in the consumer installation in order to reduce the electricity costs. In this sense, the system calculates monthly energy consumption and recommend an efficient manner to use the apparatus. The system exhibits energy consumption graphs per equipment besides a report with a home appliances ranking according to the energy consumption. The results of an analysis of representative residential consumer demonstrate the system's effectiveness in estimating energy consumption beyond to calculate the value of the electric bill. The adoption of new behaviors based on system's recommendations leads to an effective reduction of expenses with the electric bill, promoting an optimized use of the electric grids.

Keywords: Electricity consumption, energy efficiency, Software solutions, Smart systems based learning. 